or omitted; bibliographies are given at the ends of several chapters in which such proofs can be found. The typography and diagrams are excellent; few misprints are to be found.

Chapter 1 deals with complex variable theory, including integration by the residue method and conformal transformations. In Chapter 2, Fourier series and integrals, and their application to electric circuits, are discussed. Chapter 3 covers vectors, vector products, divergence, curl, gradient, and scalar and vector potential. Matrices are discussed in Chapter 4, with applications to the theory of quadripoles and specific examples, to the solution of algebraic equations, and to the determination of free oscillations of networks. In Chapter 5, tensors and curvilinear co-ordinates and their applications are considered, with particular reference to crystals and other anisotropic media. Chapter 6 deals with methods, including approximation methods, for solving ordinary and partial differential equations. Chapter 7 has separate sections dealing with hyperbolic func. tions, sine and cosine integral functions, the error functions and Legendre functions. Chapter 8 deals with operational calculus; applications to the solution of differential and integral equations as well as to electrical problems are considered. Chapter 9 deals with the theory of probability, and includes a section edited by M. Blanc-Lapierre on aleatory functions. Applications to errors of measurement and the method of least squares are discussed.

Many will have more than sufficient mathematical ability to understand the parts of this book relevant to their work. For such people the fundamental groundwork has been covered for an amazingly large number of topics, with full discussion of appropriate numerical examples. Once the reader has mastered this fundamental groundwork, he is provided with sufficient knowledge for making further progress by himself. The book can therefore be thoroughly recommended both as a text-book and especially as a reference book that will be needed continually by the practical man.

J. W. HEAD<smiles></smiles>

\section{RADAR FOR STUDENTS}

\section{A Textbook of Radar}

A Collective Work by the Staff of the Radiophysics Laboratory, CoundiN for Scientific and Industrial Research, Autralla. JPp. viii $+579+31$ plates. (London: Chof 6 man ang Fall, Ltd., 1948.) 50s. net. $7 \mathrm{HE}$ actix develo phent of radar dates from about 1935, avd pintilinabout 1941 most of the important work was done in reat Britain. After 1941 the con. tributions to Alued radar equipment made by the Unit d States, Canada and Australia increased, until eventually the contributions of the United States alone outstripped those of Britain. The work in Australia was carried out by the Radiophysics Laboratory of the Council for Scientific and Industrial Research in co-operation with industry. This Laboratory was established in 1939, and although small in comparison with those in the United States and in Great Britain, it made significant contributions to Allied equipment. For example, the service in the Pacific of the Australian air-warning radar, Type $L W / A W$, bears witness to the value of its work.

"A Textbook of Radar" is a collective work by the staff of the Radiophysics Laboratory, and it is a synthesis of the subject as seen in about 1946. The book contains twenty chapters, thirty-one plates and an index. The text is admirably illustrated by diagrams and photographs, and is well printed and free from errors.

The first chapter is an introduction, which is mostly historical. The second is called "Fundamentals", and deals with the basic principles and equations which govern a radar system, together with a discussion of propagation. The next thirteen chapters form three-quarters of the book and deal with the components. They discuss the magnetron, the pulsed operation of triode power oscillators, pulse modulators, microwave transmission and cavity resonators, aerials and aerial duplexing, receivers and their associated oscillators and mixers, display circuits and automatic ranging circuits. These thirteen chapters are contributed by twelve different authors. The next four chapters discuss the application of radar systems to uses on land, at sea and in the air. The final chapter deals with navigation.

A text-book such as this must balance three main elements, the history, the problems of radar systems viewed as a whole, and the discussion of instrumental techniques. A proper balance is made difficult by the rapid, recent and largely secret development of the subject. This book achieves a fairly good balance; however, the lapse of three years and the appearance of specialized volumes on technique suggest that more space might well have been given to the funda. mentals of radar systems at the expense of the discussion of technique.

The historical element in the book is excellent and is largely contributed by Dr. E. G. Bowen, who is well known as an original member of Sir Robert Watson-Watt's pioneer team. It is gratifying, in view of the usual exclusive importance given to microwaves, to find an adequate place given to the contribution of 'metre-wave' radar to the War.

The chapters dealing with technique leave 'metrewave' problems to other works and concentrate on microwaves. They are clear, fundamental and of sufficiently high standard to be valuable to advanced students and to radar workers. With so many authors, the breadth and depth of treatment varies, and one could comment and criticize many points. For example, the treatment of cavity resonators and of the transient response of receivers are both lengthy and well illustrated. On the other hand, radomes are not dealt with, and angle data transmission systems get only glancing references.

The chapter on "Fundamentals" by J. L. Pawsey is excellent and might well have been expanded. The remaining chapters on radar systems and on navigation are very condensed. The one dealing with ground radar is well illustrated and interesting. It omits, however, mention of moving-target indicators, presumably because of secrecy at the time of writing, and also fails to discuss adequately the very important subject of how ground radar information is displayed and used. The chapter on navigation is parochial in its treatment of civil aviation and gives an interesting description of some Australian equipments. It fails to give adequate space to the application of radar to the merchant marine.

Nevertheless, as a single volume on the subject for the advanced student, research worker or practising engineer, this book has few competitors. In a larger library on radar and electronics it will be valued, not only as a text-book, but also as another account of radar from one of the war-time Allied laboratories.

R. HaNBURY Brown 